\title{
Dynamic needle tip positioning versus the angle-distance technique for ultrasound- guided radial artery cannulation in adults: a randomized controlled trial
}

\author{
Bing Bai ${ }^{1+}$, Yuan Tian ${ }^{1+}$, Yuelun Zhang ${ }^{2}$, Chunhua Yu ${ }^{1 *}$ (D) and Yuguang Huang ${ }^{1}$
}

\begin{abstract}
Background: Ultrasound guidance can increase the success rate and reduce the incidence of complications of arterial cannulation. There are few studies on the utility of the dynamic needle tip positioning (DNTP) technique versus the angle-distance (AD) technique for ultrasound-guided radial arterial cannulation in adult surgical patients. We assessed and compared the success rates and incidences of complications of these two short-axis out-of-plane techniques.
\end{abstract}

Methods: A total of 131 adult surgical patients were randomized into DNTP and AD groups to undergo ultrasound-guided radial artery cannulation. The primary outcome was first-pass success without posterior wall puncture. The secondary outcomes included the first-pass success rate, 10-min overall success rate, cannulation time, posterior wall puncture, and the number of skin punctures.

Results: The first-pass success rates without posterior wall puncture were $53.8 \%$ in the DNTP group and $44.6 \%$ in the $A D$ group $(R R=1.22,95 \% \mathrm{Cl}: 0.86-1.72 ; P=0.26)$. The cannulation time was significantly longer $(P=0.01)$ in the DNTP group [79.65 (54.3-109.4) seconds] than in the AD group [47.6 (24.9-103.8) seconds]. The posterior wall puncture rate was significantly lower $(P=0.002)$ in the DNTP group $(29.2 \%)$ than in the AD group $(56.1 \%$; $R R=0.56$, 95\% Cl: 0.42-0.82).

Conclusions: There were no significant differences in the first-pass success rate, with or without arterial posterior wall puncture, or in the 10-min overall success rate between the DNTP and AD groups. However, the cannulation time was longer and the posterior wall puncture rate was lower in the DNTP group.

Trial registration: The trial was registered at www.clinicaltrials.gov (No: NCT03656978). Registered 4 September 2018.

Keywords: Catheterization, Radial artery, Ultrasonography

\footnotetext{
* Correspondence: ychh@pumch.cn

${ }^{+}$Bing Bai and Yuan Tian contributed equally to this work.

'Department of Anaesthesiology, Peking Union Medical College Hospital,

Chinese Academy of Medical Sciences and Peking Union Medical College, Beijing 100730, China

Full list of author information is available at the end of the article
}

(c) The Author(s). 2020 Open Access This article is licensed under a Creative Commons Attribution 4.0 International License, which permits use, sharing, adaptation, distribution and reproduction in any medium or format, as long as you give appropriate credit to the original author(s) and the source, provide a link to the Creative Commons licence, and indicate if changes were made. The images or other third party material in this article are included in the article's Creative Commons licence, unless indicated otherwise in a credit line to the material. If material is not included in the article's Creative Commons licence and your intended use is not permitted by statutory regulation or exceeds the permitted use, you will need to obtain permission directly from the copyright holder. To view a copy of this licence, visit http://creativecommons.org/licenses/by/4.0/. The Creative Commons Public Domain Dedication waiver (http://creativecommons.org/publicdomain/zero/1.0/) applies to the data made available in this article, unless otherwise stated in a credit line to the data. 


\section{Background}

Arterial cannulation is a common invasive procedure that enables beat-to-beat blood pressure monitoring, frequent blood sampling and the assessment of fluid responsiveness in operating rooms and intensive care units $[1,2]$. Different sites can be chosen for arterial cannulation, but the radial artery is most commonly used due to its superficial course, its dual blood supply to the hand, and a low rate of complications [3]. Complications from arterial cannulation include infection, hematoma, vasospasm and nerve injury $[4,5]$. Studies have shown that the success rate is higher and the incidence of related complications lower when performing this technique with ultrasound guidance than when performing it with traditional palpation [6-8].

Using ultrasound, vascular structures can be viewed in two orientations: short-axis/out-of-plane or long-axis/inplane [9]. The short-axis view has the advantages of providing better visualization of the surrounding structures and easier imaging. The traditional short-axis technique, that is, the angle-distance (AD) method, is accurate in terms of positioning and is convenient for novices to master. Earlier studies have suggested that using the AD technique can significantly improve the success rate of cannulation. However, despite the advantages of the short-axis technique, such as accurate positioning of the puncture point and visualization of the relevant perivascular structures, posterior wall puncture of the target artery might not be avoided $[10,11]$.

In recent years, studies have reported that a modified ultrasound-guided short-axis technique, i.e., the dynamic needle tip positioning (DNTP) technique, is superior to the palpation technique in both adult and neonate surgical patients [6, 12]. Researchers have found that when using the modified technique, after seeing the blood return, the probe needs to be moved further to ensure that the tube is in the arterial lumen before the next step is performed, and this might reduce the risk of posterior wall penetration $[8,13,14]$. However, this modified method requires frequent movement of the probe, which requires more operator experience and might take more time [15]. Notably, no studies have compared the success and complication rates of the DNTP technique and the AD technique. Therefore, we believe that it is necessary to compare the two techniques. We compared these two techniques and determined the strengths and weaknesses of each to provide a reference for the clinical choice of a specific technique.

\section{Methods}

\section{Ethics and registration}

This trial was approved by the institutional review board for human studies at Peking Union Medical College Hospital, Peking Union Medical College (Institutional
Review Board \#zs1338), and written informed consent was obtained from all subjects participating in the trial. The trial was registered at www.clinicaltrials.gov prior to patient enrolment (number: NCT03656978; principal investigator: Y.T.; date of registration: September 3, 2018).

\section{Randomization and blinding}

The trial adheres the applicable CONSORT guidelines. Patients scheduled to undergo elective surgery and clinically require arterial cannulation at Peking Union Medical College Hospital (Beijing, China) were enrolled from September 2018 to February 2019. The inclusion criteria for this trial were patients undergoing elective surgery clinically requiring arterial cannulation, older than 18 years and with ASA level I-IV. The exclusion criteria were contraindications for peripheral arterial puncture or catheterization, a blocked or embolized target vessel determined by ultrasound assessment, and patient refusal (Fig. 1). The anesthesiologist who conducted the cannulation procedure knew the allocation of the patients. The patients were blinded to the allocation. The statistician did not know the allocation. Enrolled patients were randomized by computer-generated numbers provided in sealed opaque envelopes to either the DNTP or AD group. The seal of the envelope was broken just before the cannulation procedure. The operator was an experienced senior anesthesiology resident in our department, who had already conducted the DNTP and $\mathrm{AD}$ techniques in over 100 patients each and was equally skilled in the 2 methods. An intention-to-treat analysis was used in this trial.

\section{Intervention and control}

In the operating room, after securing intravenous access and routine monitoring using electrocardiography, pulse oximetry, and noninvasive blood pressure, patients were given $1 \mathrm{mg}$ of midazolam iv and $50 \mu \mathrm{g}$ of fentanyl iv as well as oxygen through a mask. The wrist of the patient was extended over a roll to raise the arm and stabilized by taping it to an arm board. An ultrasound machine (Navi s; Wisonic, Inc., Shenzhen, Guangdong, China) and probe (L15-4NB; Wisonic, Inc.) were used before the procedure based on randomization to obtain an image of the radial artery and acquire measurements. Sterile techniques, including sterile gloves, operating towels, and ultrasonic probe covers, were used for arterial catheterization. After sterile skin preparation, ultrasound-guided arterial cannulation was performed using a 20-gauge intravenous catheter (BD Angiocath, Becton Dickinson Infusion Therapy Systems, Inc., Sandy, Utah, USA). The patients were administered $0.2 \mathrm{ml}$ of $2 \%$ lidocaine for local anesthesia at the insertion site before the cannulation procedure. 

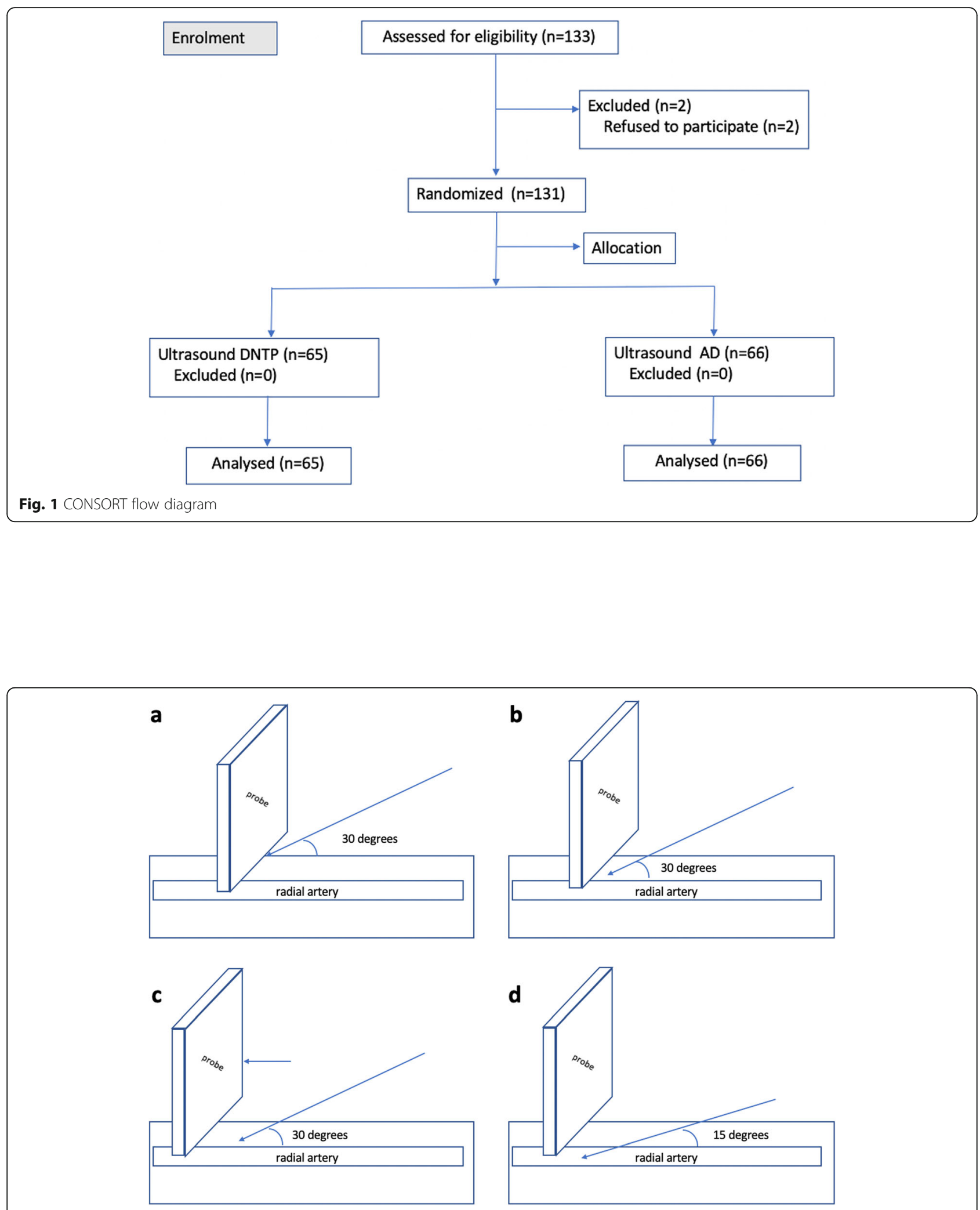

Fig. 2 Diagrams showing how to advance the needle into the radial artery using the DNTP technique. a, Obtain an image of the target artery and adjust the insertion point and orientation of the needle. b. Puncture the target artery, and observe the needle in the artery lumen. $\mathbf{c}$, Advance the ultrasound probe away from the needle until the hyperechoic needle is no longer observed. $\mathbf{d}$, Advance the needle and catheter until the very tip is seen again, and repeat these steps until the tip is observed in the artery lumen 
For both techniques, a timer was started when the operator was ready to use the needle to pass through the skin and stopped when an arterial waveform appeared. If the procedure lasted more than $10 \mathrm{~min}$, the operator was forced to stop and free to use any technique until success was achieved. In that case, the cannulation was recorded as $600 \mathrm{~s}$. The research team defined first-pass success as the successful acquisition of a regular waveform on the screen after just one pass through the skin.

For the DNTP technique, the probe was placed to view the out-of-plane radial artery and moved to place the artery in the center of the ultrasound screen. Then, the needle was inserted at the point at which the middle mark of the probe contacted the skin and advanced through the skin at an angle of approximately 30 degrees until the tip was seen on the screen (Fig. 2). The probe was moved along the long-axis of the target artery away from the insertion point until the tip just disappeared from the screen. Then, we advanced the needle and catheter until the tip was just seen again. These steps were repeated until the tip was observed in the artery lumen. The cannula was then inserted into the artery, and the needle was removed.

For the $\mathrm{AD}$ technique, the probe was placed to view the short-axis plane of the target artery and moved to place the artery in the center of the ultrasound screen. Then, the distance from the surface of the skin to the anterior wall of the artery was measured. The needle was inserted at the point at which the middle mark of the probe contacted the skin and advanced through the skin. Since an initial angle of 45 degrees between the needle and skin was used for puncture, the distance between the point of insertion and the central point of the probe was approximately equal to the distance from the surface of the skin to the anterior wall of the artery (Fig. 3). Then, the needle was advanced until blood appeared in the hub. The needle angle was decreased slightly while the catheter was advanced slightly. The catheter was advanced into the target artery only if blood continued to flow into the hub. If insertion failed or the target was missed, the needle insertion direction was adjusted according to the position of the needle tip.

\section{Outcomes}

The following baseline data were collected for each patient: sex, age, height, weight, body mass index, systolic blood pressure, diastolic blood pressure, heart rate just before the procedure, history of hypertension, diabetes, coronary heart disease or smoking, surgery type, radial artery diameter and radial artery depth. The primary outcome was first-pass success without posterior wall puncture. The secondary outcomes included the firstpass success rate, the 10-min overall success rate, the cannulation time, posterior wall puncture, and the number of skin punctures. First-pass success without posterior wall puncture was defined as successful catheterization without vascular damage to the posterior wall. First-pass success was defined as successful

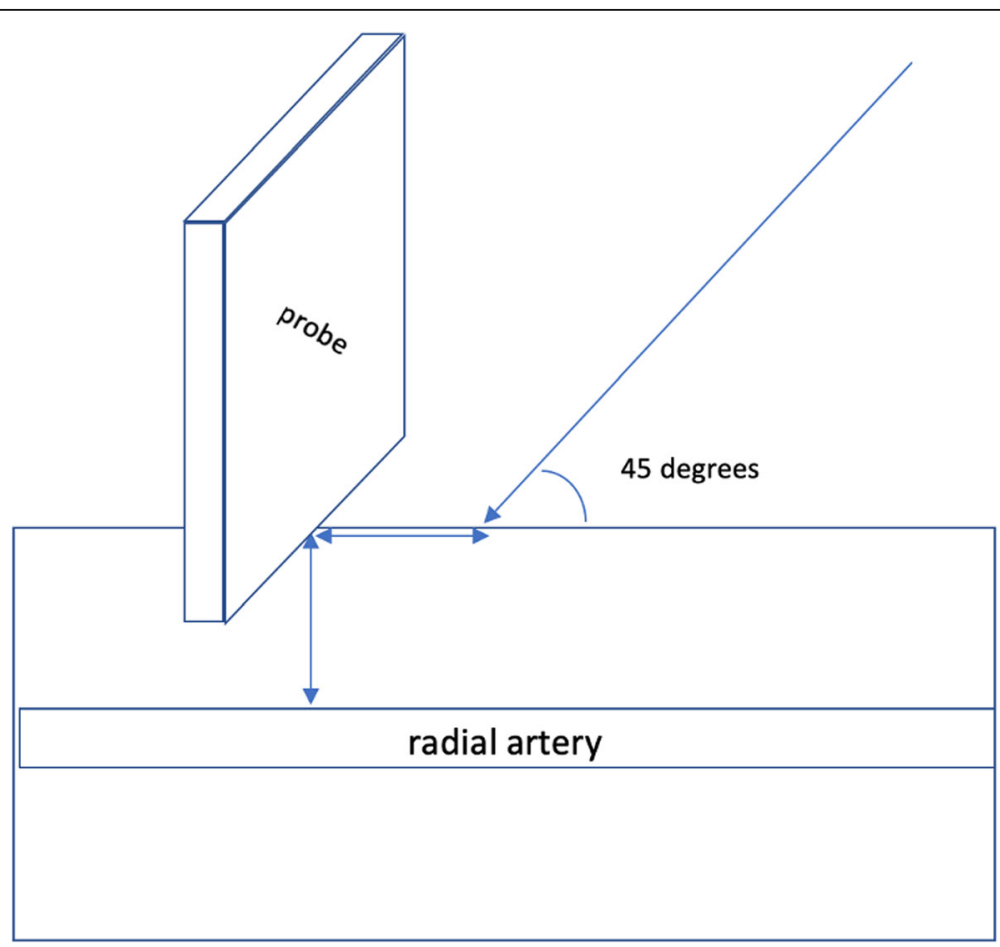

Fig. 3 Insertion point and orientation of the needle when using the AD technique 
catheterization on the first attempt regardless of vascular damage to the posterior wall. Overall success was defined as successful catheterization regardless of vascular damage to the posterior wall and without a limit on the number of punctures. The cannulation time was defined as the interval between skin contact with the probe and confirmation of the arterial waveform on the monitor. Posterior wall puncture of the radial artery was considered when the operator saw the needle passing the posterior wall or blood backflow appeared then disappeared while needle advancing. The number of skin punctures refers to the number of skin punctures that occurred during the whole cannulation procedure.

\section{Statistical analysis}

The baseline characteristics of the participants are described using descriptive statistics. The clinical and demographic characteristics of patients in the two groups were compared using the t-test, the chi-square test, and nonparametric rank tests, and a $P$ value less than 0.05 was considered to indicate a statistically significant imbalance between the groups. When analyzing the primary outcome, imbalanced baseline characteristics were adjusted using logistic regression analysis. The primary outcome, first-pass success without posterior wall puncture, was compared using the chi-square test and relative risk with the corresponding calculated 95\% confidence intervals. Similarly, the secondary outcomes, which included first-pass success, 10-min overall success, posterior wall puncture and the number of skin punctures, were also compared using the chi-square test. The median cannulation time was compared using the Mann-Whitney U test. Statistical analysis was conducted on an intention-to-treat basis using SPSS (version 25.0; IBM Corp). A two-sided $P$ value less than 0.05 was considered statistically significant.

\section{Sample size calculation}

In our preliminary trial, the first-pass success rate was $77 \%$ with the DNTP technique and $53 \%$ with the AD technique. To compare the $53 \%$ first-attempt success rate in the $\mathrm{AD}$ group with the $77 \%$ rate in the $\mathrm{DNTP}$ group, with a power of $80 \%$ and a two-sided type I error rate of 0.05 , we needed to enroll 61 patients per group.

Table 1 Baseline characteristics of all patients enrolled in the trial

\begin{tabular}{|c|c|c|c|}
\hline & \multicolumn{2}{|l|}{ Technique } & \multirow[t]{2}{*}{$P$ Value } \\
\hline & DNTP $(n=65)$ & $\mathrm{AD}(n=66)$ & \\
\hline Male, n (\%) & $39(60.0)$ & $40(66.6)$ & 0.94 \\
\hline Age, y & $59 \pm 14$ & $58 \pm 13$ & 0.68 \\
\hline Height, cm & $166 \pm 8$ & $169 \pm 8$ & 0.11 \\
\hline Weight, kg & $68 \pm 9$ & $70 \pm 6$ & 0.16 \\
\hline Body mass index, $\mathrm{kg} / \mathrm{m}^{2}$ & $25 \pm 3$ & $25 \pm 3$ & 0.83 \\
\hline ASA level & & & 0.80 \\
\hline$|-| \mid$ & 17 & 16 & \\
\hline III-IV & 48 & 50 & \\
\hline Systolic blood pressure, mmHg & $143 \pm 20$ & $149 \pm 19$ & 0.09 \\
\hline Diastolic blood pressure, $\mathrm{mmHg}$ & $74 \pm 21$ & $79 \pm 23$ & 0.23 \\
\hline Heart rate, bpm & $69 \pm 26$ & $70 \pm 23$ & 0.72 \\
\hline Hypertension, n (\%) & $38(59)$ & $33(50)$ & 0.33 \\
\hline Diabetes, n (\%) & $33(51)$ & $31(47)$ & 0.66 \\
\hline Coronary heart disease, n (\%) & $31(48)$ & $35(53)$ & 0.54 \\
\hline Smoking, n (\%) & $38(59)$ & $39(59)$ & 0.94 \\
\hline Surgery type, n (\%) & & & 0.45 \\
\hline Heart surgery & $41(63.1)$ & $46(69.7)$ & \\
\hline General surgery & $8(12.3)$ & $7(10.6)$ & \\
\hline Orthopaedic surgery & $2(3.1)$ & $5(7.6)$ & \\
\hline Urological surgery & $8(12.3)$ & $6(9.1)$ & \\
\hline Vascular surgery & $6(9.2)$ & $2(3.0)$ & \\
\hline Radial artery diameter, mm & $2.30 \pm 0.50$ & $2.38 \pm 0.50$ & 0.36 \\
\hline Median radial artery depth (IQR), mm & $3.10(2.10-4.00)$ & $2.70(2.10-3.80)$ & 0.40 \\
\hline
\end{tabular}

Abbreviation: IQR interquartile range 
Considering a dropout rate of $10 \%$, a total of 136 patients were planned for enrolment.

\section{Results}

A total of 136 patients were recruited and screened for eligibility in the trial from September 2018 to February 2019. Five of the patients were excluded from the trial because they refused to sign the consent form. Finally, we randomized 131 patients who underwent a nonemergent operation and required radial arterial cannulation in the final analysis, as shown in Fig. 1. There were 79 $(60.3 \%)$ males and 52 (39.7\%) females, and the average age was $58.27 \pm 13.26$ years. There were 65 patients in the DNTP group and 66 patients in the AD group. The basic characteristics are shown in Table 1, and there were no significant differences between the two groups.

\section{Primary outcomes}

Regarding the primary outcome, the first-pass success rate without posterior wall puncture was $53.8 \%$ in the DNTP group and $44.6 \%$ in the AD group $(R R=1.22$, 95\% CI 0.86-1.72, $P=0.26$; Table 2).

\section{Secondary outcomes}

Regarding the secondary outcomes, the first-pass success rate was $53.8 \%$ in the DNTP group and $59.1 \%$ in the AD group ( $\mathrm{RR}=0.90,95 \% \mathrm{CI} 0.63-1.27, P=0.54$; Table 2). The overall success rate was $86.2 \%$ in the DNTP group and $80.3 \%$ in the AD group ( $\mathrm{RR}=1.22$, 95\% CI $0.82-$ $1.81, P=0.37$; Table 2). The time to cannulate the artery was significantly longer in the DNTP group, at 79.65 (54.3-109.4) seconds, than in the AD group, at 47.6 (24.9-103.8) seconds, $(P=0.01$; Table 2$)$. The posterior wall puncture rate was significantly lower in the DNTP group (29.2\%) than in the $\mathrm{AD}$ group (56.1\%) $(\mathrm{RR}=0.56$, 95\% CI 0.42-0.82, $P=0.002$; Table 2). There was no significant difference between the two groups in the number of skin punctures (Table 2).

\section{Discussion}

This trial demonstrates that as ultrasound-guided outof-plane radial artery cannulation techniques, the DNTP and $\mathrm{AD}$ techniques are both effective in adult surgical patients. However, although there was no significant difference in the success rate of the two methods, each method has its own advantages and disadvantages. Compared with the AD method, the DNTP method reduced the incidence of posterior wall puncture but also required additional time. In clinical practice, we sometimes encounter patients with poor coagulation and are worried that the arterial puncture will break through the posterior wall and cause complications, such as hematoma. According to our results, it may be more appropriate to use the DNTP technique in these cases. In other cases, such as patients in shock, we are most concerned about catheterizing the artery as soon as possible. In such instances, we may recommend the AD method. Our results could serve as a reference for the above clinical issues.

Recently, many clinical studies have demonstrated the superiority of ultrasound-guided arterial catheterization. Several meta-analyses have shown that ultrasoundguided methods are superior to palpation methods in adults, children, and even newborns [16-19]. According to previous comparisons of out-of-plane ultrasoundguided versus palpation for arterial catheterization, the traditional out-of-plane ultrasound-guided technique, similar to our AD technique, has a first-pass success rate

Table 2 Trial results

\begin{tabular}{|c|c|c|c|c|}
\hline & \multicolumn{2}{|l|}{ Technique } & \multirow[t]{2}{*}{$P$ Value } & \multirow{2}{*}{$\begin{array}{l}\text { Relative Risk } \\
(95 \% \mathrm{Cl})\end{array}$} \\
\hline & DNTP $(n=65)$ & $\mathrm{AD}(n=66)$ & & \\
\hline \multicolumn{5}{|l|}{ Primary outcome } \\
\hline First-pass success without posterior wall puncture, \% & $35(53.8)$ & $29(44.6)$ & $0.26^{\mathrm{a}}$ & $1.22(0.86-1.72)$ \\
\hline \multicolumn{5}{|l|}{ Secondary outcomes } \\
\hline First-pass success, $\%$ & $35(53.8)$ & $39(59.1)$ & $0.54^{\mathrm{a}}$ & $0.90(0.63-1.27)$ \\
\hline 10-min overall success, $\%$ & $56(86.2)$ & $53(80.3)$ & $0.37^{\mathrm{a}}$ & $1.22(0.82-1.81)$ \\
\hline Median cannulation time (IQR), s & $79.7(54-109)$ & $47.6(25-104)$ & $0.007^{b}$ & \\
\hline Posterior wall puncture, $\%$ & $19(29.2)$ & $37(56.1 \%)$ & $0.002^{\mathrm{a}}$ & $0.56(0.42-0.82)$ \\
\hline Number of skin punctures & & & $0.86^{a}$ & \\
\hline 1 & 50 & 52 & & \\
\hline 2 & 3 & 1 & & \\
\hline$\geq 3$ & 12 & 13 & & \\
\hline
\end{tabular}

Abbreviation: $I Q R$ interquartile range

${ }^{a}$ Chi-square test

${ }^{\mathrm{b}}$ Mann-Whitney $U$ test 
between 53 and $78 \%[11,15]$. Recently, researchers reported that a modified technique, the DNTP technique, has a first-pass success rate of $82 \%$ [6], suggesting that the success rate was higher for the DNTP technique than the traditional AD method. However, there have been no direct comparisons of these two out-of-plane methods. More importantly, ultrasound-guided arterial catheterization has been recommended for its higher success rate and lower complication rate. However, to the best of our knowledge, no published reports have evaluated both the success and complication rates concurrently. Importantly, posterior wall puncture may cause problems, such as hematoma [9]. Therefore, we used the rate of first-pass success without posterior wall puncture as our primary outcome to direct compare these two methods.

We found that there were no significant differences in the rate of first-pass success without posterior wall puncture. This is different from our preliminary trial results, which could be attributed to the following: first, we might have overestimated the difference between the two methods in the preliminary trial; second, the sample size might not have been sufficient. Compared to the results of previously reported studies, in our trial, the first-pass success rates and overall success rates were different for both methods. We think that these differences are more representative of the experience ranges of different operators at each research center than of differences between the methods, as our operator had already conducted DNTP and $\mathrm{AD}$ techniques in over 100 patients each before the trial and was equally skilled in the 2 methods.

Ultrasound-guided vascular cannulation includes an in-plane technique and out-of-plane technique [13]. Using in-plane technology, the needle trajectory from needle insertion to catheter placement is clearly visible, which seems to be effective for reducing the incidence of posterior vessel wall puncture [9]. However, this technology requires the operator to be very skilled at ultrasound technology, which is more dependent on experience and can be difficult for novices to master; on the other hand, given that the long axis is subject to slice-thickness artifacts, due to the measurable thickness of the ultrasound beam itself, the cannula in the long axis can sometimes appear to be in the same plane as the extremely small radial artery, even when the cannula has not been successfully inserted into the artery [20, 21]. Therefore, we prefer the out-of-plane technique.

Arterial catheterization consists of two steps: the tip of the needle enters the blood vessel, and the catheter is then placed into the blood vessel [6]. Out-of-plane ultrasound guidance methods can assist in accurately locating the target artery and penetrating the anterior wall of the artery. The subsequent step is similar to that used in traditional palpation. In the DNTP method, the needle tip needs to be observed in the artery lumen, and this may explain why compared to the $\mathrm{AD}$ method, the DNTP method significantly reduces the chance of posterior wall perforation. Another concern of using ultrasound is the time required. Our trial shows that compared to the AD method, the DNTP method significantly reduced the probability of penetrating the posterior wall of the artery but also takes more time to complete the procedure. We speculate that the reason for this difference is not only that the DNTP method requires more time for ultrasound probe use but also that the time required is related to the operator's experience with the ultrasound probe. As the operator gains experience with ultrasound, the difference in the time between the two methods might decrease.

In fact, ultrasound-guided arterial catheterization involves many details and techniques, such as the previously reported "follow the tip" [14] and "saline injection" methods [20]. From this perspective, the DNTP and AD methods are not static but are constantly evolving. In addition, technological developments in ultrasound machines and probes can have a significant impact on the operator, especially the novice. We should pay attention to these details and techniques, continue to improve them, and finally find the ideal, standardized method to achieve the goal of truly benefiting patients with less harm.

There are several limitations to our trial. First, the ultrasound-guided arterial cannulation procedures were performed by a senior resident; therefore, one should be cautious about applying our data to other practitioners. Second, we may have overestimated the difference in our primary outcome between the two methods in the preliminary trial, and if the sample size had been larger, the results might have been different.

In conclusion, there was no significant difference in the rate of first-pass success without posterior wall puncture, the first-pass success rate or the overall success rate between the DNTP and AD methods for ultrasound-guided radial artery cannulation. The DNTP method reduced the incidence of posterior wall puncture of the target artery but required more time. These conclusions could serve as a reference for anesthesiologists when choosing which method to use for arterial cannulation in a specific clinical situation.

\section{Conclusions}

There were no significant differences in the first-pass success rate, with or without arterial posterior wall puncture, or in the 10-min overall success rate between the DNTP and AD groups. However, the cannulation time was longer and the posterior wall puncture rate was lower in the DNTP group than in the AD group. The appropriate technique should be applied depending on the specific clinical situation. 


\section{Abbreviations}

DNTP: Dynamic needle tip positioning; AD: Angle-distance; ASA: American Society of Anesthesiologists; IQR: Interquartile range.

\section{Acknowledgements}

The authors wish to thank their colleagues at the Department of Anaesthesiology of the Peking Union Medical College Hospital for their support.

\section{Authors' contributions}

BB contributed to the design of the study, administration of the study, data collection, data analysis, and manuscript preparation. YT contributed to the design of the study, administration of the study, data collection, data analysis, and manuscript preparation. $\mathrm{YZ}$ contributed to the design of this study and data analysis. CY contributed to the original idea for the study, design of the study, and manuscript preparation. YH contributed to the design of this study and administration of the study. All authors read and approved the final manuscript.

\section{Funding}

No funding was obtained for this study.

\section{Availability of data and materials}

The datasets used and analyzed during the current study are available from the corresponding author on request.

\section{Ethics approval and consent to participate}

This trial was approved by the institutional review board for human studies at Peking Union Medical College Hospital, Peking Union Medical College (Institutional Review Board \#zs1338), and written informed consent was obtained from all subjects participating in the trial.

\section{Consent for publication}

Not applicable.

\section{Competing interests}

The authors declare that they have no competing interests.

\section{Author details}

${ }^{1}$ Department of Anaesthesiology, Peking Union Medical College Hospital, Chinese Academy of Medical Sciences and Peking Union Medical College, Beijing 100730, China. ${ }^{2}$ Medical Research Center, Peking Union Medical College Hospital, Chinese Academy of Medical Sciences and Peking Union Medical College, Beijing 100730, China.

Received: 6 June 2020 Accepted: 8 September 2020

Published online: 14 September 2020

\section{References}

1. Ye P, Tan Y, Ye M, Li S, Bai L, Liu L. A novel method for ultrasound-guided radial artery cannulation in neonates by trainee anaesthesiologists: a randomised controlled trial. Eur J Anaesthesiol. 2020;37:91-7.

2. Wang J, Lai Z, Weng $X$, Lin $Y$, Wu G, Su J, et al. Modified long-axis in-plane ultrasound technique versus conventional palpation technique for radial arterial cannulation: a prospective randomized controlled trial. Medicine (Baltimore). 2020;99:e18747.

3. Nam K, Jeon Y, Yoon S, Kwon SM, Kang P, Cho YJ, et al. Ultrasound-guided radial artery cannulation using dynamic needle tip positioning versus conventional long-axis in-plane techniques in cardiac surgery patients: a randomized, controlled trial. Minerva Anestesiol. 2020;86:30-7.

4. Zhefeng Q, Luo C, Zhang L, Li X, He H, Chi P. Application of optimized ultrasonic localization system for radial artery puncture by intern doctors: a randomized trial. Med Sci Monit. 2019;25:1566-71.

5. Yu Y, Lu X, Fang W, Liu X, Lu Y. Ultrasound-guided artery cannulation technique versus palpation technique in adult patients in pre-anesthesia room: a randomized controlled trial. Med Sci Monit. 2019;25:7306-11.

6. Kiberenge RK, Ueda K, Rosauer B. Ultrasound-guided dynamic needle tip positioning technique versus palpation technique for radial arterial cannulation in adult surgical patients: a randomized controlled trial. Anesth Analg. 2018;126:120-6.
7. White L, Halpin A, Turner M, Wallace L. Ultrasound-guided radial artery cannulation in adult and paediatric populations: a systematic review and meta-analysis. Br J Anaesth. 2016;116:610-7.

8. Berk D, Gurkan Y, Kus A, Ulugol H, Solak M, Toker K. Ultrasound-guided radial arterial cannulation: long axis/in-plane versus short axis/out-of-plane approaches? J Clin Monit Comput. 2013;27:319-24.

9. Song IK, Choi JY, Lee JH, Kim EH, Kim HJ, Kim HS, et al. Short-axis/out-ofplane or long-axis/in-plane ultrasound-guided arterial cannulation in children: a randomised controlled trial. Eur J Anaesthesiol. 2016;33:522-7.

10. Shiver S, Blaivas M, Lyon M. A prospective comparison of ultrasound-guided and blindly placed radial arterial catheters. Acad Emerg Med. 2006;13:1275-9.

11. Levin PD, Sheinin O, Gozal Y. Use of ultrasound guidance in the insertion of radial artery catheters. Crit Care Med. 2003;31:481-4.

12. Liu L, Tan Y, Li S, Tian J. "Modified dynamic needle tip positioning" shortaxis, out-of-plane, ultrasound-guided radial artery cannulation in neonates: a randomized controlled trial. Anesth Analg. 2019;129:178-83.

13. Abdalla UE, Elmaadawey A, Kandeel A. Oblique approach for ultrasoundguided radial artery catheterization vs transverse and longitudinal approaches, a randomized trial. J Clin Anesth. 2017;36:98-101.

14. Goh G, Tan C, Weinberg L. Dynamic ultrasound-guided, short axis, out-ofplane radial artery cannulation: the 'follow the tip' technique. Anaesth Intensive Care. 2013:41:431-2.

15. Ueda K, Bayman EO, Johnson C, Odum NJ, Lee JJ. A randomised controlled trial of radial artery cannulation guided by doppler vs palpation vs ultrasound. Anaesthesia. 2015;70:1039-44.

16. Tang L, Wang F, Li Y, Zhao L, Xi H, Guo Z, et al. Ultrasound guidance for radial artery catheterization: an updated meta-analysis of randomized controlled trials. PLoS One. 2014;9:e111527.

17. Gao YB, Yan JH, Gao FQ, Pan L, Wang XZ, Lv CJ. Effects of ultrasoundguided radial artery catheterization: an updated meta-analysis. Am J Emerg Med. 2015;33:50-5.

18. Pacha HM, Alahdab F, Al-Khadra Y, Idris A, Rabbat F, Darmoch F, et al. Ultrasound-guided versus palpation-guided radial artery catheterization in adult population: a systematic review and meta-analysis of randomized controlled trials. Am Heart J. 2018;204:1-8.

19. Bhattacharjee S, Maitra S, Baidya DK. Comparison between ultrasound guided technique and digital palpation technique for radial artery cannulation in adult patients: an updated meta-analysis of randomized controlled trials. J Clin Anesth. 2018:47:54-9.

20. Nakayama Y, Nakajima Y, Sessler DI, Ishii S, Shibasaki M, Ogawa S, et al. A novel method for ultrasound-guided radial arterial catheterization in pediatric patients. Anesth Analg. 2014;118:1019-26.

21. Goldstein A, Madrazo BL. Slice-thickness artifacts in gray-scale ultrasound. J Clin Ultrasound. 1981:9:365-75.

\section{Publisher's Note}

Springer Nature remains neutral with regard to jurisdictional claims in published maps and institutional affiliations.

Ready to submit your research? Choose BMC and benefit from:

- fast, convenient online submission

- thorough peer review by experienced researchers in your field

- rapid publication on acceptance

- support for research data, including large and complex data types

- gold Open Access which fosters wider collaboration and increased citations

- maximum visibility for your research: over $100 \mathrm{M}$ website views per year

At $\mathrm{BMC}$, research is always in progress.

Learn more biomedcentral.com/submission 